\title{
Application of Artificial Intelligence in the Diagnosis of Eosinophilia
}

S. Martha Merlyn, S. Shiney Valentina, Sachidanand Singh, J. Jannet Vennila and Atul Kumar*

Department of Bioinformatics, Karunya University, Coimbatore, Tamil Nadu, India

\begin{abstract}
Artificial intelligence is a branch of computer science capable of analysing complex medical data. Their potential to exploit meaningful relationship with in a data set can be used in the diagnosis, treatment and predicting outcome in many clinical scenarios. The task of medical diagnosis is a complex one, considering the level vagueness and uncertainty management, especially when the disease has multiple symptoms. Fuzzy logic controller (FLC) was used to design a system for the diagnosis of eosinophilia. Eosinophilia is a common disease which is prevalent in people. High eosinophilic count in blood is an evidence for prevalence of eosinophilia. It is caused by both external and internal factors. The remedial measures for eosinophilia should be taken at the earliest, as the effect for it can vary from mild to severe. The design is based on Mamdani-style inference system which is very good for the representation of human reasoning and effective analysis. The implementation is done using MATLAB fuzzy logic tools. The effectiveness of FLC depends on the rules formed and interpretation of surface data. The performance of our FLC was predicted about $82.5 \%$ and a minimum error was obtained as $10.0 \%$.
\end{abstract}

Keywords: Eosinophilia; Eosinophilic count; Fuzzy logic; Fuzzy inference system

\section{Introduction}

Eosinophils are a type of white blood cell. They contain particles filled with chemicals that fight off infections and play a role in your body's immune response. Normally our blood doesn't have a large number of eosinophils. Our body may produce more of them in response to allergic disorders, inflammation of the skin, and parasitic infections. They can also increase in response to some infections or to some bone marrow disorders [1]. In some conditions, the eosinophils can move outside the bloodstream and into organs and tissues. Treatment of the problem depends on the cause.

Diagnosis of the condition of eosinophilia is via blood count, but diagnosis of the underlying cause varies depending on the suspected condition. Treatment is determined by the causative condition. Eosinophilia can be idiopathic (primary) or secondary to another disease [3]. In the United States, allergic or atopic diseases are the most common causes, especially those of the respiratory systems. In the developing world, parasites are the most common cause. A parasitic infection of nearly any bodily tissue can cause eosinophilia.

Hypereosinophilia is a subcategory of idiopathic eosinophilia; diagnosis requires documentation of both sustained eosinophilia (absolute eosinophil count $\geq 1500$ cells/ $\mu \mathrm{L}$ for at least 6 months. An increase in eosinophils, i.e., the presence of more than 500 eosinophils/ micro litre of blood is called an eosinophilia. The normal concentration is between 0 and $0.5 \times 10^{9}$ eosinophils per litre of blood. Eosinophilia can be reactive (in response to other stimuli such as allergy or infection) or non reactive [2]. The release of interleukin 5 by $\mathrm{T}$ cells, mast cells and macrophages stimulates the production of eosinophils.

Alternative knowledge acquisition approaches based on fuzzy logic have recently attracted considerable attention. It is essential to realize that fuzzy logic uses truth degrees as a mathematical model of the vagueness phenomenon. Fuzzy logic resembles human reasoning in its use of approximate information and uncertainty to generate decisions [4]. An approach to uncertainty that combines real values $[0 \ldots 1]$ and logic operations is based on the ideas of fuzzy set theory and fuzzy set membership function.

This work presents the application of fuzzy logic to automate knowledge acquisition for diagnosis of Eosinophilia. Fuzzy logic is applied for the diagnosis of eosinophilia on knowledge base which consists of a data base and a rule base. The advantage of these methods over other approaches is that they can produce good results in situations where data is imprecise, noisy and inconsistent.

\section{Materials and Methods}

Fuzzy logic controller (FLC), a successful application of Zadeh's fuzzy set theory; [5] is a potential tool for dealing with uncertainty and imprecision. Thus, the knowledge of a doctor can be modeled using an FLC [6]. The performance of an FLC depends on its knowledge base which consists of a data base and a rule base. It is observed that the performance of an FLC mainly depends on its rule base and optimizing the membership function distributions stored in the data base is a fine tuning process [7].

The process of knowledge acquisition has been long recognized as one of the most complex and laborious tasks in the construction of expert systems. In some situations, lack of available expertise can make development of such systems impossible. Eliciting knowledge from experts encounters numerous obstacles. Although the experts are highly skilled in solving problems in their domain, they often have difficulty in articulating their knowledge or sometimes even in understanding their own decision making processes.

\section{Designing of fuzzy inference system}

Fuzzy inference is a method that interprets the values in the input and based on user defined Rules, assigns values to the output. For developing diagnosing tool for Eosinophilia, data is required; the data

*Corresponding author: Atul Kumar, Department of Bioinformatics, Karunya University, Coimbatore, Tamil Nadu, India, Tel: +919488523540; E-mail: atul.0298@gmail.com

Received December 13, 2010; Accepted December 27, 2010; Published December 29, 2010

Citation: Merlyn SM, Valentina SS, Singh S, Vennila JJ, KumarA(2010)Application of Artificial Intelligence in the Diagnosis of Eosinophilia. J Health Med Informat 1:103. doi:10.4172/2157-7420.1000103

Copyright: (c) 2010 Merlyn SM, et al. This is an open-access article distributed under the terms of the Creative Commons Attribution License, which permits unrestricted use, distribution, and reproduction in any medium, provided the original author and source are credited. 
should be capable of representing the whole disease and also the severity of the disease. Basically the data is sign and symptoms of patients, some medical report etc. Hence, data was collected from Jeyasekaran Medical Trust, Nagercoil from which 150 patients were analysed. By consulting doctor and by analysing the data of the patients four symptoms were finalised as the inputs for diagnosing the eosinophilia. The four symptoms which are relevant and mostly used for the diagnosis of eosinophilia were used as an input in fuzzy inference system as shown below. Based on these symptoms presence or absence of eosinophilia was predicted as an output.

\section{Physical symptoms}

1. Redness

2. Frequent wheezing.

3. Breathlessness due to asthma.

4. Parasitic infections symptoms - abdominal pain, diarrhoea, fever, cough and rashes.

5. Pneumonia.

6. Anaemia

\section{Medical tests}

7. 6. Absolute Eosinophilic Count

8. 7. Differential Count

\section{Algorithm for the fuzzy inference system for diagnosis of eosinophilia}

For the four symptoms four input variables and one output variable "Eosinophilia" was created. The four symptoms which are considered here as an input variable for diagnostic system are crucial and mainly considered for the detection and diagnosis of Eosinophilia. The four

\begin{tabular}{|l|l|l|}
\hline Membership Function & Type & Params \\
\hline Normal & Trimf & {$\left[\begin{array}{lll}1 & 5 & 10\end{array}\right]$} \\
\hline Mild & Trimf & {$\left[\begin{array}{lll}7 & 13 & 20\end{array}\right]$} \\
\hline Moderate & Trimf & {$\left[\begin{array}{lll}16 & 23 & 30\end{array}\right]$} \\
\hline Severe & Trimf & {$\left[\begin{array}{lll}25 & 62 & 100\end{array}\right]$} \\
\hline
\end{tabular}

Table 1: Membership Function for Input Variable differential count.

\begin{tabular}{|c|c|c|}
\hline Membership Function & Type & Params \\
\hline Normal & Trimf & {$\left[\begin{array}{llll}0 & 500 & 1000\end{array}\right]$} \\
\hline Mild & Trimf & {$\left[\begin{array}{llll}600 & 1300 & 2000\end{array}\right]$} \\
\hline Moderate & Trimf & {$\left[\begin{array}{lllll}1500 & 3500 & 5000]\end{array}\right.$} \\
\hline Severe & Trimf & {$\left[\begin{array}{llll}5000 & 7500 & 10000\end{array}\right]$} \\
\hline
\end{tabular}

Table 2: Membership Function for Input Variable absolute eosinophilic count.

\begin{tabular}{|l|l|l|}
\hline Membership Function & Type & Params \\
\hline Normal & Trimf & {$\left[\begin{array}{lll}15 & 16.5 & 18\end{array}\right]$} \\
\hline Mild & Trimf & {$\left[\begin{array}{lll}13 & 14.5 & 16\end{array}\right]$} \\
\hline Moderate & Trimf & {$\left[\begin{array}{lll}11 & 12.5 & 14\end{array}\right]$} \\
\hline Severe & Trimf & {$\left[\begin{array}{lll}8 & 10 & 12\end{array}\right]$} \\
\hline
\end{tabular}

Table 3: Membership Function for Input Variable Haemoglobin Count

\begin{tabular}{|l|l|l|}
\hline Membership Function & Type & Params \\
\hline Normal & Trimf & {$\left[\begin{array}{lll}0 & 1.5 & 3\end{array}\right]$} \\
\hline Mild & Trimf & {$\left[\begin{array}{lll}2.5 & 4 & 5.5\end{array}\right]$} \\
\hline Moderate & Trimf & {$\left[\begin{array}{lll}5 & 6.5 & 8\end{array}\right]$} \\
\hline Severe & Trimf & {$\left[\begin{array}{lll}7 & 8.5 & 10\end{array}\right]$} \\
\hline
\end{tabular}

Table 4: Membership Function for Input Variable Redness.

\begin{tabular}{|c|c|c|}
\hline Membership Function & Type & Params \\
\hline No Eosinophilia & Trimf & {$\left[\begin{array}{lll}0 & 25 & 50\end{array}\right]$} \\
\hline Eosinophilia & Trapmf & {$\left[\begin{array}{lllllll}50 & 75 & 100\end{array}\right]$} \\
\hline
\end{tabular}

Table 5: Membership Function for Input Variable of type Eosinophilia.

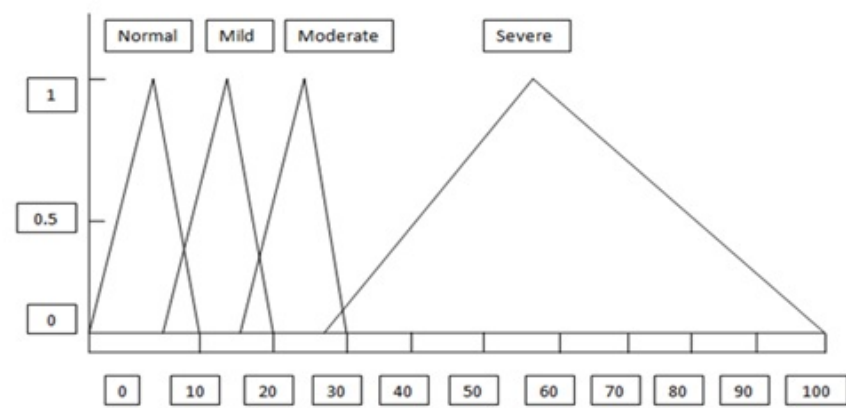

Figure 1: Membership Function Plot for Input Variable Differential Count.

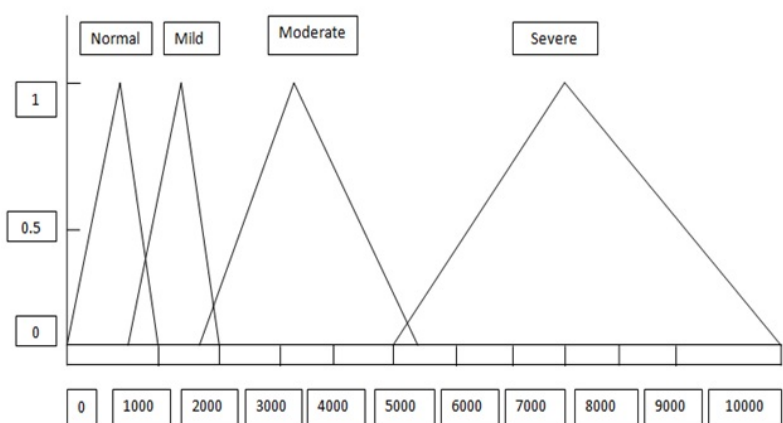

Figure 2: Membership Function Plot for Input Variable Absolute Eosinophilic Count.

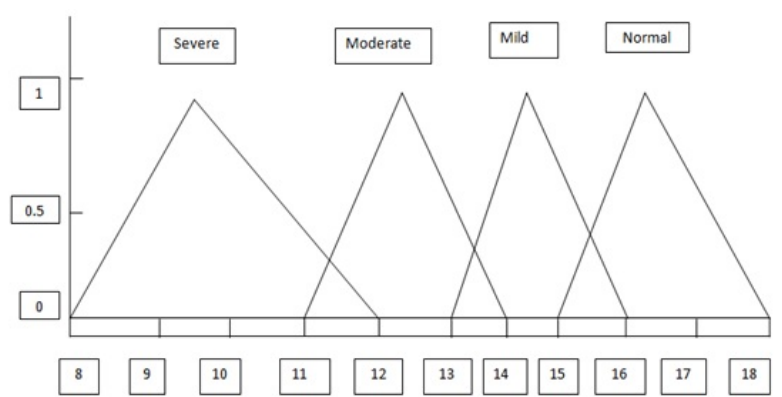

Figure 3: Membership Function Plot for Input Variable Haemoglobin Count.

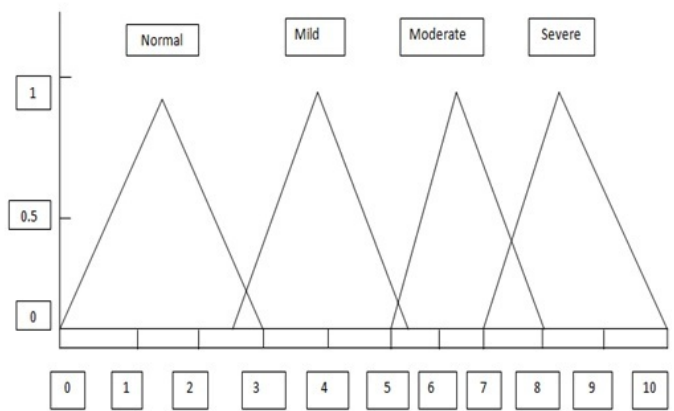

Figure 4: Membership Function Plot for Input Variable Redness.

input variables (Symptoms) are divided into 4 Membership Functions based on the frequency and percentage value of the symptoms. The Membership Function and Membership Function plot for four input variables are shown in the following Tables (1-5) and Figures (1-5). 
Based on the descriptions of the input and output variables 30 rules were constructed by selecting an item in each input and output variable box and one Connection (AND). None was chosen as one of the variable qualities to exclude any of the variables from a given rule. The weight was specified to unity (1). Table 6 shows the rule base for the Eosinophilia inference system.

Based on these rules the roadmap of the whole fuzzy inference

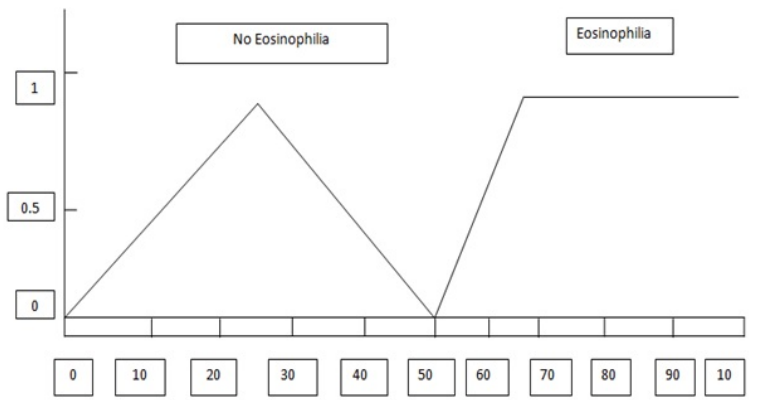

Figure 5: Membership Function Plot for Input Variable Type of Eosinophilia

\begin{tabular}{|c|c|c|c|c|c|}
\hline S.NO. & DC & AEC & HB & RN & OP \\
\hline 1 & Normal & Moderate & Severe & normal & EP \\
\hline 2 & Severe & Normal & Mild & Severe & $\mathrm{NE}$ \\
\hline 3 & Moderate & Severe & Moderate & mild & EP \\
\hline 4 & Normal & Mild & Normal & Moderate & $\mathrm{NE}$ \\
\hline 5 & Normal & severe & None & Normal & EP \\
\hline 6 & Normal & Normal & Moderate & Severe & $\mathrm{NE}$ \\
\hline 7 & mild & moderate & None & mild & EP \\
\hline 8 & Mild & Normal & Normal & Severe & $\mathrm{NE}$ \\
\hline 9 & Mild & Severe & Moderate & Normal & EP \\
\hline 10 & Mild & Mild & Severe & Normal & $\mathrm{NE}$ \\
\hline 11 & Moderate & Moderate & Mild & Severe & EP \\
\hline 12 & Moderate & Normal & Normal & Mild & $\mathrm{NE}$ \\
\hline 13 & Moderate & Mild & Moderate & Mild & $\mathrm{NE}$ \\
\hline 14 & Severe & Moderate & Severe & Severe & EP \\
\hline 15 & Severe & Severe & Normal & Mild & EP \\
\hline 16 & Severe & Mild & Moderate & Moderate & $\mathrm{NE}$ \\
\hline 17 & Normal & Mild & None & Severe & $\mathrm{NE}$ \\
\hline 18 & Normal & Moderate & None & Normal & EP \\
\hline 19 & Normal & Severe & None & Mild & EP \\
\hline 20 & Mild & Moderate & Moderate & None & EP \\
\hline 21 & Mild & Severe & Severe & None & EP \\
\hline 22 & Severe & Normal & Mild & None & $\mathrm{NE}$ \\
\hline 23 & Severe & Mild & Normal & None & $\mathrm{NE}$ \\
\hline 24 & Normal & Moderate & Moderate & mild & EP \\
\hline 25 & Severe & Severe & Mild & Mild & EP \\
\hline 26 & Moderate & Severe & Normal & moderate & EP \\
\hline 27 & Mild & Normal & Moderate & Moderate & $\mathrm{NE}$ \\
\hline 28 & Mild & Normal & Severe & severe & $\mathrm{NE}$ \\
\hline 29 & Mild & Moderate & Severe & Severe & EP \\
\hline 30 & Mild & Severe & None & None & EP \\
\hline 31 & Mild & Severe & Mild & normal & EP \\
\hline 32 & Moderate & Mild & Severe & None & $\mathrm{NE}$ \\
\hline 33 & Moderate & Severe & None & None & EP \\
\hline 34 & Moderate & Normal & Severe & Severe & $\mathrm{NE}$ \\
\hline 35 & Moderate & Moderate & None & None & EP \\
\hline 36 & Moderate & Mild & None & None & $\mathrm{NE}$ \\
\hline 37 & Severe & Severe & None & Mild & EP \\
\hline 38 & Severe & Normal & Moderate & Moderate & $\mathrm{NE}$ \\
\hline 39 & Severe & Moderate & Moderate & Moderate & EP \\
\hline 40 & Severe & Mild & Normal & Moderate & $\mathrm{NE}$ \\
\hline 41 & Severe & Severe & Moderate & Mild & EP \\
\hline 42 & Moderate & Severe & Mild & Normal & EP \\
\hline 43 & Moderate & Severe & Severe & Mild & EP \\
\hline 44 & Moderate & Moderate & Severe & Severe & EP \\
\hline 45 & Severe & Moderate & Mild & Normal & EP \\
\hline
\end{tabular}

Table 6: Rule base for Eosinophilia Inference System in which DC- Differential count, AEC- Absolute Eosinophilic Count, HB- Haemoglobin, RN- Redness, OPOutput, NE- No Eosinophilia, EP- Eosinophilia.

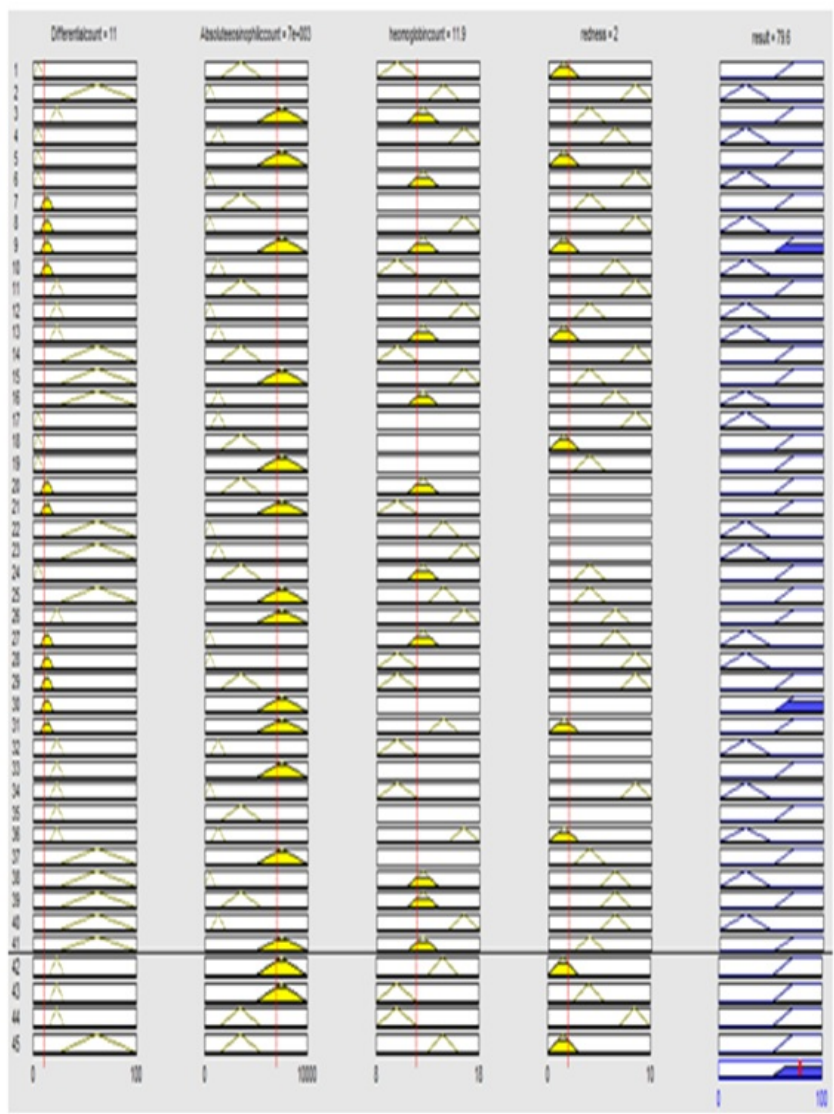

Figure 6: Rule Viewer for Eosinophilia Inference System

system is shown in Figure 6. Rule Viewer acts as a diagnostic, can show which rules is active, or how individual membership function shapes are influencing the results. It displays a roadmap of the whole fuzzy inference process. In Figure 6 the five plots across the top of the figure represent the antecedent and consequent of the first rule. Each rule is a row of plots, and each column is a variable. The rule numbers are displayed on the left of each row.

There are 226 plots nested in a single figure window. The four plots across the top of the figure represent the antecedent and consequent of the first rule. Each rule is a row of plots, and each column is a variable. The rule numbers are displayed on the left of each row. The first four columns of plots (the yellow plots) show the membership functions referenced by the antecedent, or the if-part of each rule. The fifth column of plots (the blue plots) shows the membership functions referenced by the consequent, or the then-part of each rule. The plots which are blank in the if-part of any rule correspond to the characterization of none for the variable in the rule. The last plot in the fifth column of plots represents the aggregate weighted decision for the given inference system. This decision will depend on the input values for the system. The defuzzified output is displayed as a bold vertical line on this plot. The variables and their current values are displayed on top of the columns. In the lower left, there is a text field Input for entering specific input values.

\section{Results and Discussion}

Based on the rules the inference system calculated the type and severity of Eosinophilia by following AND connection and then defuzzyfying the generated output using the centroid method. The 
AND connection was used to perform logical operation (Table 7). In fuzzy logic the truth of any statement is matter of degree so the AND connection performed a min operation, i.e. resolved the statement $\mathrm{A}$ AND $B$, where $A$ and $B$ are limited to the range $(0,1)$ by using the function $\min (\mathrm{A}, \mathrm{B})$.

The truth table was converted to a plot of two fuzzy sets applied together to create one fuzzy set. Figure 7 displays how the operations work over a continuously varying range of truth values $\mathrm{A}$ and $\mathrm{B}$ according to the defined fuzzy operations.

\section{Defuzzification of the output}

As much as fuzziness helps the rule evaluation during the intermediate steps, the final desired output for each variable is generally a single number. However, the aggregate of a fuzzy set encompasses a range of output values, and so must be defuzzified in order to resolve a single output value from the set. The defuzzification method used here was the centroid calculation, which returns the centre of area under the curve (Figure 8).

The defuzzified value was calculated based on the following equation:

$$
\mathrm{d}_{\mathrm{CA}}(\mathrm{C})=\frac{\int_{-c}^{c} \mathrm{C}(\mathrm{z}) \mathrm{zdz}}{\int_{-c}^{c} \mathrm{C}(\mathrm{z}) \mathrm{zdz}}
$$

Where $d_{C A}(C)$ is the defuzzified value and $C$ is the Membership Function.

\begin{tabular}{|c|c|c|}
\hline A & B & Min (A, B) \\
\hline 0 & 0 & 0 \\
\hline 0 & 1 & 0 \\
\hline 1 & 0 & 0 \\
\hline 1 & 1 & 1 \\
\hline
\end{tabular}

Table 7: Logical operation performed by AND Connection in Fuzzy Logic.

\begin{tabular}{|c|c|c|c|c|c|}
\hline Val & DC & AEC & HB & RN & OP \\
\hline Val.1 & 11 & 7200 & 11.9 & 2 & 88.23 \\
\hline Val.2 & 6 & 1000 & - & 8.5 & 83.33 \\
\hline Val.3 & 16 & 7200 & 11.2 & - & 93.75 \\
\hline Val.4 & 25.6 & 500 & 13.5 & - & 71.42 \\
\hline Val.5 & 4 & 10000 & - & 3.5 & 75.75 \\
\hline Val.6 & 4 & 500 & 11.5 & 8.5 & 83.62 \\
\hline Val.7 & 11 & 6200 & 13.5 & 2 & 89.76 \\
\hline Val.8 & 14 & 700 & 8.7 & 8 & 83.33 \\
\hline Val.9 & 25.6 & 5000 & 14.5 & 2 & 84.33 \\
\hline Val.10 & 25.9 & 1200 & 17.6 & 6.5 & 71.4 \\
\hline
\end{tabular}

Table 8: Validation results of Eosinophilia in which Val- validation of patient, DCDifferential count, AEC- Absolute Eosinophilic Count, HB- Haemoglobin, RNRedness, OP- Output.

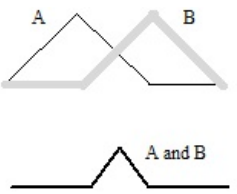

Figure 7: Membership Function Plot for Input Variable Type of Eosinophilia

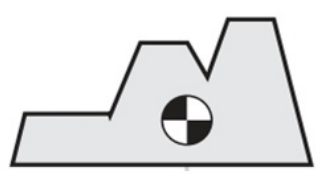

Figure 8: Defuzzification of the aggregate output.
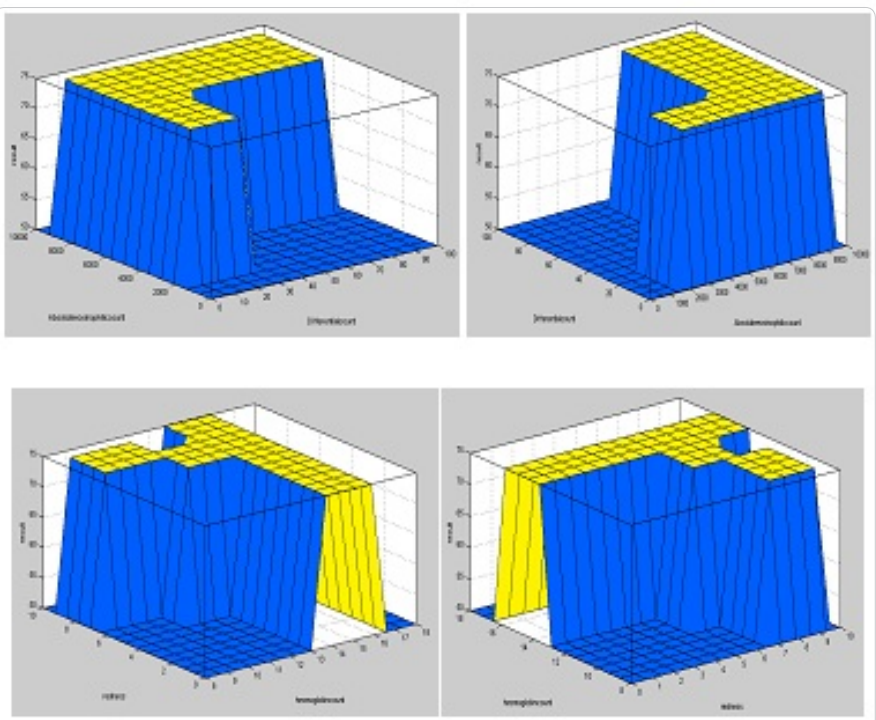

Figure 9: Plot of an output surface map for the system based on the rules.

Based on the AND operation every rule was examined for a given set of Input values and the rule which satisfied the operational logic was used to generate the output for the Inference system. The output given by each rule was aggregated and then defuzzified using centroid calculation to generate a single output which was a single number representing the severity of Arthritis.

\section{Testing of the arthritis fuzzy inference system}

The effectiveness of the developed Fuzzy Inference System was tested by providing ten sets of field data. Results of the manually constructed FLC was found to be correct when compared with the field data output. It happened because the rule base of the constructed FLC was designed after a careful study of the effects of different inputs on the output, i.e., grade of the disease. Based on the output of the FLC which is expressed on a scale of $0-100$, decision can be taken on the severity of Eosinophilia for a particular set of input symptoms. Table 8 shows the testing data and the generated output by the Fuzzy Inference System.

The entire span of the output set based on the entire span of the input set is shown in Figure 9. It displays the dependency of one of the outputs on anyone or two of the inputs i.e., it shows the plot of an output surface map for the system based on the rules defined in rule base.

\section{Conclusion}

This work was undertaken with the aim to design a system for the diagnosis of Eosinophilia diagnosis and its severity using Fuzzy Logic so that common people who suspects little bit of pain in there joints, may use this system and get the result on the diagnosis and severity of Eosinophilia which will be helpful to guide him to take proper curative measures before the severity increases. The results obtained from the systems reveal that the diagnostic system for Eosinophilia is giving expected results. The efficacy of the system developed is to be endorsed by the doctors in the field observation. Hence, afterwards the system can be made open to all through Internet. With the consultation of the doctors, the curative measures suggested by them can be made available with the system.

\section{Acknowledgement}

The authors are profoundly grateful to "Dr. Deva Prasad", Jeyasekaran Nursing home and Trust, Nagercoil, who helped a lot to collect the data, 
Citation: Merlyn SM, Valentina SS, Singh S, Vennila JJ, Kumar A (2010) Application of Artificial Intelligence in the Diagnosis of Eosinophilia. J Health Med Informat 1:103. doi:10.4172/2157-7420.1000103

Page 5 of 5

which helped us to significantly improve the diagnostic system. We express our deep sense of gratitude to Mr. Sachidanand Singh, lecturer Department of Bioinformatics for his invaluable guidance, support and encouragement.

\section{References}

1. Ayalew T (2005) Blood Eosinophilia: A New Paradigm in Disease Classification Diagnosis, and Treatment. Mayo Clin Proc 80: 75-83.

2. Polat K, Gunes S (2007) An expert system approach based on principal component analysis and adaptive neuro-fuzzy inference system to diagnosis of diabetes disease. Digital Signal Processing 17: 702-710.

3. Linkens DA, Nyongesa $\mathrm{HO}$ (1996) Learning systems in intelligent control: an appraisal of fuzzy, neural and genetic algorithm control applications. IEE Proc Control Theory Appl 143: 367-386.

4. Zadeh LA (1965) Fuzzy sets. Inform Contr 8: 338-353.

5. Mamdani EH, Assilian S (1975) An experiment in linguistic synthesis with a fuzzy logic controller. International Journal of Man-Machine Studies 7: 1-13.

6. Pratihar DK, Deb K, Ghosh A (1990) A genetic-fuzzy approach for mobile robot navigation among moving obstacles. Int J Approx Reason 20: 145-172.

7. Shin MD, Park MD, Kim MD, Kee MD (2007) Eosinophilic Enteritis Presenting as Intussusception in Adult. The Korean Journal of Internal Medicine 22: 13-17. 